\title{
Mitochondria directly donate their membrane to form autophagosomes during a novel mechanism of parkin-associated mitophagy
}

\author{
Katherine L Cook', David R Soto-Pantoja ${ }^{2}$, Mones Abu-Asab³, Pamela AG Clarke ${ }^{1}$, David D Roberts² \\ and Robert Clarke 1* $^{*}$
}

\begin{abstract}
Background: Autophagy (macroautophagy), a cellular process of "self-eating", segregates damaged/aged organelles into vesicles, fuses with lysosomes, and enables recycling of the digested materials. The precise origin

(s) of the autophagosome membrane is unclear and remains a critical but unanswered question. Endoplasmic reticulum, mitochondria, Golgi complex, and the plasma membrane have been proposed as the source of autophagosomal membranes.
\end{abstract}

Findings: Using electron microscopy, immunogold labeling techniques, confocal microscopy, and flow cytometry we show that mitochondria can directly donate their membrane material to form autophagosomes. We expand upon earlier studies to show that mitochondria donate their membranes to form autophagosomes during basal and drug-induced autophagy. Moreover, electron microscopy and immunogold labeling studies show the first physical evidence of mitochondria forming continuous structures with LC3-labeled autophagosomes. The mitochondria forming these structures also stain positive for parkin, indicating that these mitochondrial-formed autophagosomes represent a novel mechanism of parkin-associated mitophagy.

Conclusions: With the on-going debate regarding autophagosomal membrane origin, this report demonstrates that mitochondria can donate membrane materials to form autophagosomes. These structures may also represent a novel form of mitophagy where the mitochondria contribute to the formation of autophagosomes. This novel form of parkin-associated mitophagy may be a more efficient bio-energetic process compared with de novo biosynthesis of a new membrane, particularly if the membrane is obtained, at least partly, from the organelle being targeted for later degradation in the mature autolysosome.

Keywords: Breast cancer, Mitochondria, Autophagy, Mitophagy, Parkin, Antiestrogen resistance, Fulvestrant, Imatinib, Estrogen receptor-a

\section{Findings}

Autophagy involves the segregation of subcellular material into double membrane structures (autophagosomes) that then fuse with lysosomes (autolysosomes) wherein the cellular cargo is subsequently degraded by lysosomal hydrolases. This process facilitates the digestive degradation of aged, damaged, or unneeded organelles including mitochondria, Golgi complex, and endoplasmic reticulum [1].

\footnotetext{
*Correspondence: clarker@georgetown.edu

'Department of Oncology and Lombardi Comprehensive Cancer Center, Georgetown University Medical Center, Washington, DC 20057, USA Full list of author information is available at the end of the article
}

Understanding of the autophagic machinery has advanced; however the primary source of the phospholipid bilayer that creates the autophagosome membrane has remained unclear $[2,3]$.

The difficulty in identifying the origin of cellular material donated to form autophagosome membranes reflects the inability of specific markers for each subcellular organelle to carry over to autophagosomes. Thus, various organelles have been proposed to be autophagosome membrane donors including the plasma membrane, endoplasmic reticulum, Golgi complex, mitochondria, and even a de novo generation model $[2,3]$. The endoplasmic reticulum 
was originally implicated by studies reporting the concurrent presence of rough endoplasmic reticulum integral membrane proteins both in autophagosome membrane preparations and electron microscopy images [4,5]. However, contradictory data emerged indicating only $30 \%$ of all autophagosomes are associated with the endoplasmic reticulum, suggesting the involvement of other organelles in the formation of autophagosomes [6]. More recently, the outer mitochondrial membrane was proposed to serve as a donor source for starvation-induced autophagosome formation [7]. Time-lapse photography data suggested that the early autophagy protein ATG5 and the autophagosomal marker LC3 translocate to puncta localized on mitochondria, and that labeled outer mitochondrial membrane protein concurrently marked both autophagosomes and mitochondria in data obtained following serum starvation of a rat kidney cell line $[8,9]$. However, this study is limited because of the primary use of confocal microscopy and the general observation that localization is to be anticipated since the mitochondria are engulfed within mature autophagosomes during mitophagy. The resolution provided by electron microscopy (EM) is needed to directly show autophagosome structures, their content, and their special relationships with mitochondria; this evidence has been notably lacking. We show, for the first time, visual evidence of the contribution of mitochondrial membrane donation to autophagosome formation in both basal and drug-induced autophagy in a human breast cancer cell line. Moreover, these mitochondria donating membranes to form autophagosomes stain positive for the mitophagy-related protein parkin, suggesting a novel mechanism of mitophagy whereby the mitochondria contribute to autophagosome formation, other than being engulfed by the forming autophagosome [10].

\section{Materials and methods}

The following materials were obtained as indicated: Imatinib and ICI 182,780 (Tocris Bioscience, Ellisville, MO); penicillin and Improved Minimal Essential Medium (IMEM; Gibco Invitrogen BRL, Carlsbad, CA); bovine calf charcoal stripped serum (CCS) (Equitech-Bio Inc, Kerrville, TX); Lipofectamine RNAiMax reagent (Invitrogen); Estrogen receptor- $\alpha$ (ER) shRNA (Evrogen, Moscow, Russia); GFP-LC3 (Addgene, Cambridge, MA); EndoTracker Red, Golgi-RFP, MitoTracker-GFP, MitoTracker-RFP (Invitrogen); Cyto-ID Autophagosome detection kit (Enzo Life Sciences); LC3B and parkin antibody (Cell Signaling Technology, Danvers, MA); PINK1 and parkin siRNA (Origene, Rockville, MD).
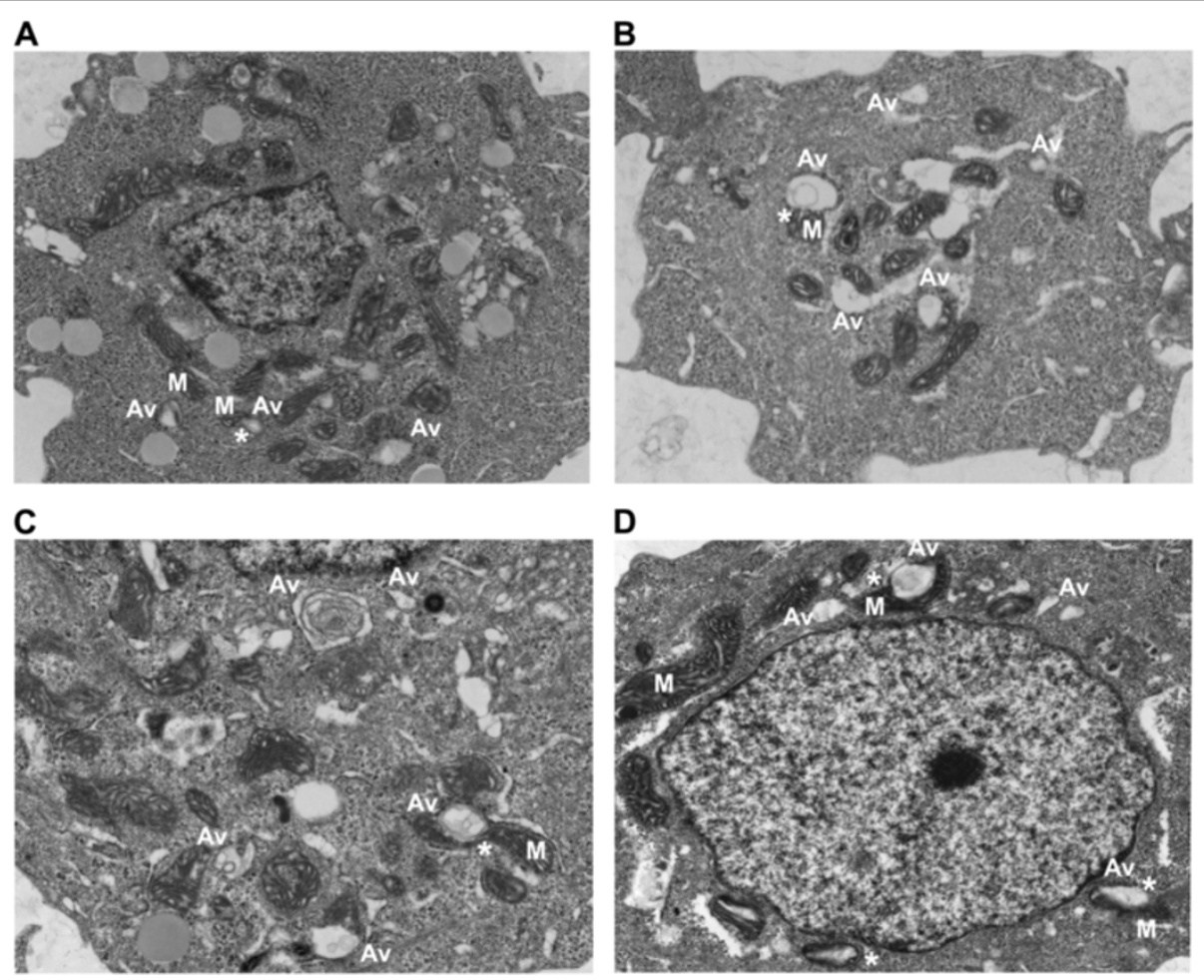

Figure 1 Drug-induced autophagy in ER + breast cancer cells exhibit elevated autophagosome formation and indicate a direct contribution of the mitochondrial membrane in autophagosome membrane development. Electron micrograph images of $\mathbf{A}$. vehicle treated LCC9 breast cancer cells. B. LCC9 cells treated with 100 nM fulvestrant for 72 hours. C. LCC9 cells transfected with estrogen receptor-a shRNA. D. LCC9 cells treated with $10 \mu \mathrm{M}$ Imatinib for 72 hours. M indicates mitochondria; Av indicates an autophagic vesicle; * indicates mitochondria-autophagosome interaction. 
LCC9 breast carcinoma cells were grown in phenol-red free IMEM media containing 5\% CCS. Cells were grown at $37^{\circ} \mathrm{C}$ in a humidified, $5 \% \mathrm{CO}_{2}: 95 \%$ air atmosphere. Cells were plated in $10 \mathrm{~cm}$ dishes and treated with $0.1 \% \mathrm{v} / \mathrm{v}$ ethanol vehicle, $100 \mathrm{nM}$ fulvestrant, or $10 \mu \mathrm{M}$ Imatinib for 72 hours, or transfected with four shRNA constructs targeting ER- $\alpha$. EM was performed as previously described [11]. Briefly, cells were pelleted and fixed with $2.5 \%$ glutaraldehyde and postfixed with $0.5 \%$ osmium tetroxide. Cells were then dehydrated and embedded in Spurs epoxy resin. Embedded cells were cut into ultrathin sections $(90 \mathrm{~nm}$ ), double-stained with uranyl acetate and lead citrate, and viewed with a Philips CM10 transmission electron microscope (Phillips Electronics). Autophagosome number and size were quantified using ImageJ software.

LCC9 cells were transfected with GFP-LC3B and control or ER $\alpha$ shRNA, $0.1 \% \mathrm{v} / \mathrm{v}$ ethanol vehicle, $500 \mathrm{nM}$ ICI, or $10 \mu \mathrm{M}$ Imatinib and with lentiviral RFP-labeled organelle trackers (endoplasmic reticulum, golgi complex, and mitochondria) for 24 hours. Cells were counterstained with DAPI and confocal microscopy was performed using an Olympus IX-70 confocal microscope (LCCC Imaging Shared Resources) to determine LC3-positive punctate formation and LC3 co-localization with different cellular organelles. LCC9 cells were treated with vehicle, serum starvation, $500 \mathrm{nM} \mathrm{ICI}, 2 \mathrm{ng} / \mathrm{mL}$ tunicamycin, transfected with ATG7 siRNA (negative control), transfected with ER $\alpha$ shRNA, transfected with parkin siRNA, or treated with $10 \mu \mathrm{M}$ Imatinib for 48 hours. Cells were incubated with MitoTracker-GFP for 24 hours prior to cell harvesting. Cells were collected and treated with a modified monodansylcadaverine. Cells were sorted by flow cytometry to quantify autophagosome and mitochondria number (LCCC Flow Cytometry Shared Resource).

The effect of mitophagy on antiestrogen responsiveness was determined by crystal violet cell density assay. Briefly, $5 \times 10^{3}$ cells/mL LCC9 cell in IMEM containing 5\% CCS were transfected with control or PINK1 siRNA and were plated in 24-well tissue culture plates. On day 1 after plating, cells were treated with varying doses of fulvestrant (10 nM-1000 nM). On day 3, medium was aspirated and cells were stained with crystal violet. Cells were permeabilized using citrate buffer and absorbance was read at $660 \mathrm{~nm}$ using a plate reader.

To confirm the effect of treatments on autophagy and subcellular localization, western blot hybridization was used to measure LC3-I/LC3-II, p62, PINK1, parkin, and COXIV. Treated cell monolayers were solubilized in lysis
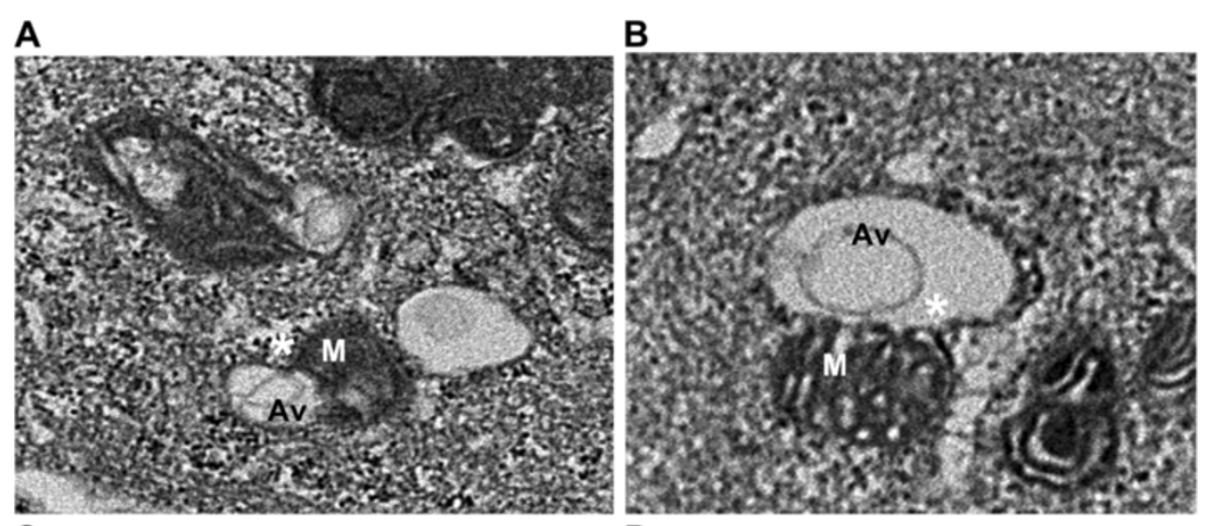

C

D
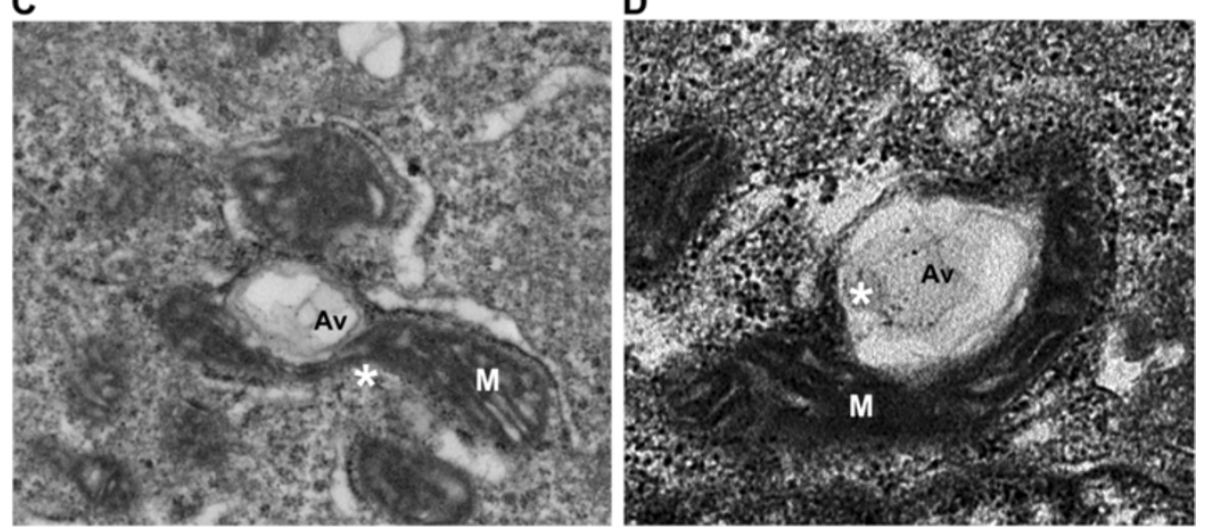

Figure 2 Mitochondria directly donate membrane material to form autophagosomes. Electron micrograph images of $\mathbf{A}$. vehicle treated LCC9 breast cancer cells. B. LCC9 cells treated with $100 \mathrm{nM}$ fulvestrant for 72 hours. C. LCC9 cells transfected with estrogen receptor-a shRNA. D. LCC9 cells treated with $10 \mu \mathrm{M}$ Imatinib for 72 hours. M indicates mitochondria; Av indicates an autophagic vesicle; * indicates mitochondria-autophagosome interaction. 
buffer, protein was measured using a standard bicinchoninic acid assay, and proteins were size fractionated by polyacrylamide gel electrophoresis and transferred to nitrocellulose membranes. Non-specific binding was blocked by incubation with Tris-buffered saline containing 5\% powdered milk and $1 \%$ Triton X-100. Membranes were incubated overnight at $4^{\circ} \mathrm{C}$ with primary antibodies, followed by incubation with polyclonal horseradish peroxidase (HRP)-conjugated secondary antibodies (1:2000) for 1 hour at room temperature. Immunoreactive products were visualized by chemiluminescence (SuperSignal Femto West, Pierce Biotechnology, Rockford, IL) and quantified by densitometry using the ImageJ digital densitometry software (http://rsbweb.nih.gov/ij/). Protein loading was visualized by incubation of stripped membranes with a monoclonal antibody to $\beta$-actin or $\beta$-tubulin (1:1000).

All data are presented as the mean \pm standard error of the mean (SEM). Statistical differences were evaluated by one way analysis of variance (ANOVA) followed by Dunnett post hoc test. The criterion for statistical significance was set at $\mathrm{p}<0.05$ prior to initiation of the study.

\section{Results and discussion}

Autophagy is often increased in response to stress, starvation, and drug treatment [12]. Antiestrogens (tamoxifen (TAM) and Fulvestrant (ICI)) induce autophagy in ER $\alpha$ expressing human breast cancer cells [12-14]. This autophagy induction is associated with cell survival, suggesting that it is a major determinant of resistance to these drugs $[15,16]$. Using the LCC9 (ER+, estrogen independent, ICI resistant, TAM cross-resistant) [17] and MCF7 (ER+, estrogen dependent, ICI and TAM sensitive) breast cancer cell line, electron microscopy was used to investigate the effect of ER knockdown and treatment with antiestrogens and other autophagy-inducing drugs on autophagosome formation. Figure 1A shows that LCC9 vehicle treated (control) cells exhibit a high level of basal autophagy as indicated by the presence of autophagosomes marked Av (autophagic vacuole). Treatment with ICI increased the formation of autophagosomes (Figure 1B), as did ER knockdown that mimics the effects of ICI on ER expression (Figure $1 \mathrm{C}$ ). Imatinib mesylate $\left(\right.$ Gleevec $^{\circ}$ ), a c-abl inhibitor previously shown to induce autophagy in chronic myeloid leukemia cells [18], was also used to stimulate further autophagosome formation (Figure 1D). Higher magnification EM images show that mitochondria directly contribute their membrane material to form autophagosomes (Figure 2). In all electron microscopy images viewed in this study, we found at least one example of mitochondrial membranes forming contiguous structures with the membranes of developing autophagosomes (as indicated by *). Quantification of autophagosome number and size are shown in Figure 3. All treatments significantly increased autophagosome number, while antiestrogen therapy and
ER shRNA treatments increased autophagosome size. The percentage of mitochondria forming these continuous vesicle-like structures was also determined (Figure 3C).

To confirm induction of autophagy using these experimental conditions, LCC9 cells were transfected with GFP-LC3 and treated either with vehicle (control), 500

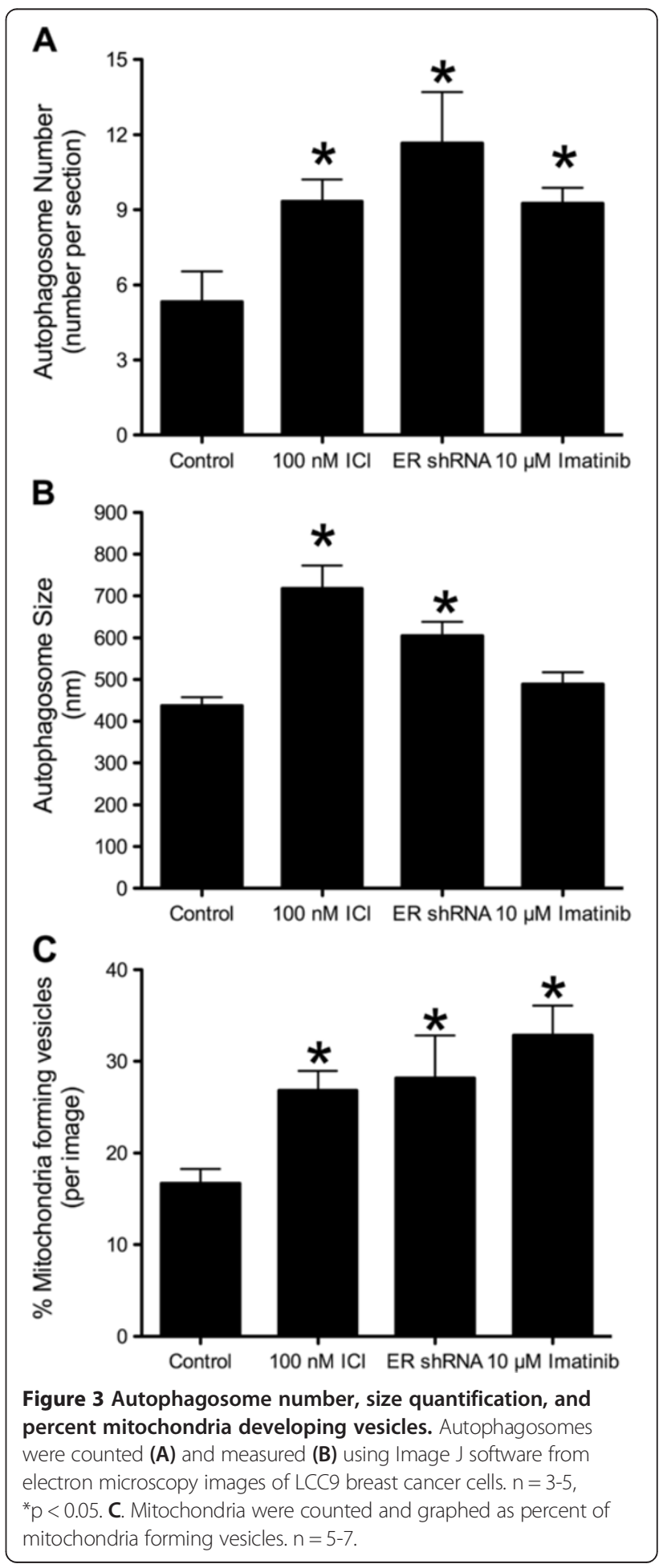


nM ICI, ER $\alpha$ shRNA, or $10 \mu \mathrm{M}$ Imatinib for 24 hours. Confocal microscopy showed that antiestrogen therapy, knockdown of $\mathrm{ER} \alpha$, and Imatinib treatment each induced LC3-positive puncta formation, demonstrating that these conditions stimulate the initiation of autophagy (Figure 4A). Furthermore, western blot hybridization on protein lysates from LCC9 cells treated with vehicle control, $100 \mathrm{nM}$ ICI, transfected with ER shRNA, or $10 \mu \mathrm{M}$ Imatinib were used to confirm treatment effects on LC3 and p62 levels (Figure 4B). All treatments increased LC3-II formation in LCC9 cells, indicating that ICI, Imatinib, and ER knockdown increased autophagosome formation. ER knockdown and ICI treatment decreased p62 levels showing that these drugs increased autophagic flux. However treatment of LCC9 cells with Imatinib increased p62, suggesting that the $\mathrm{c}-\mathrm{abl}$ inhibitor blocked autophagic flux by preventing lysosome degradation of autophagosomes. The effect of imatinib on autophagy and antiestrogen resistance will be further explored in future work. LCC9 cells were treated either with vehicle (control), or
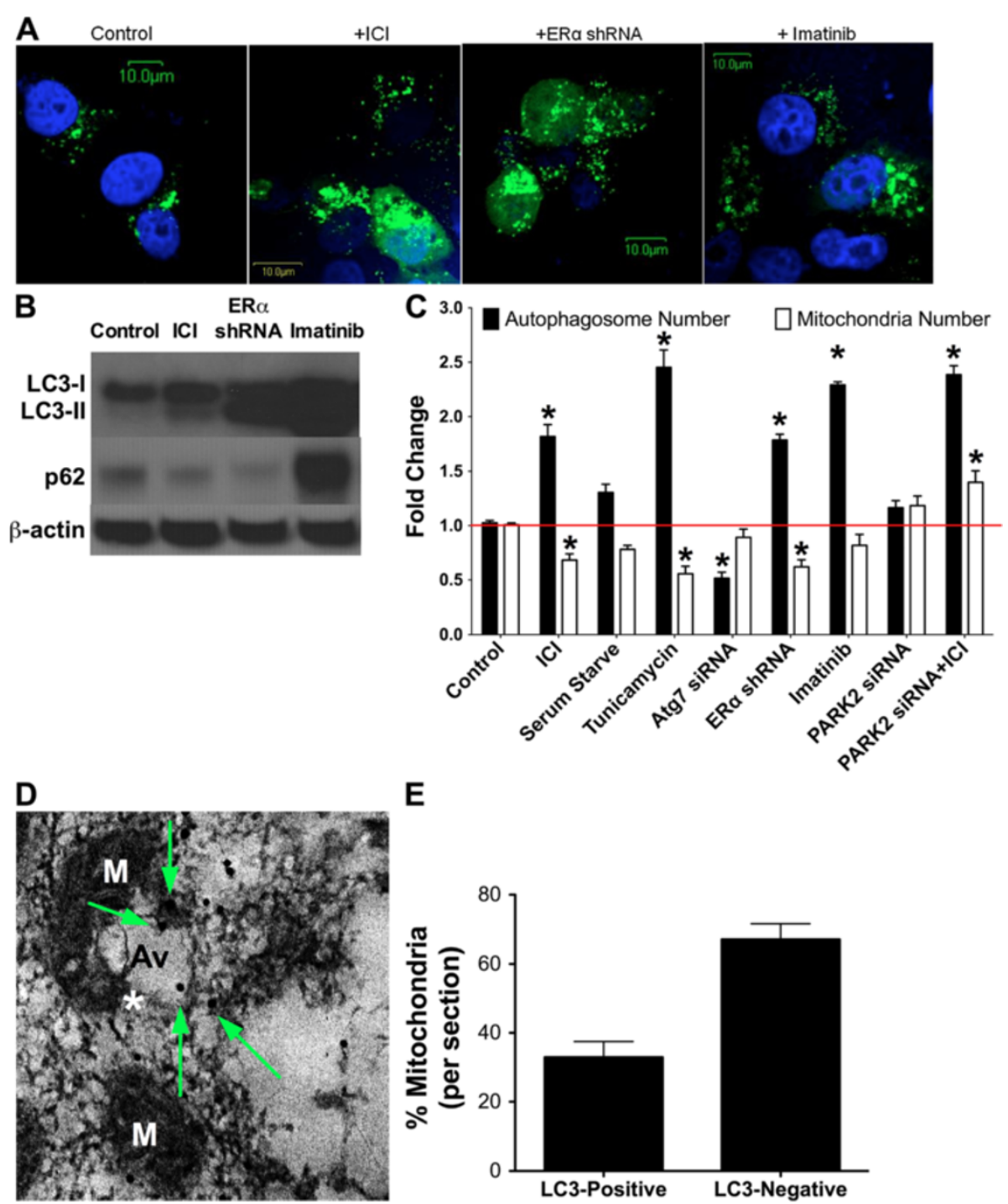

E

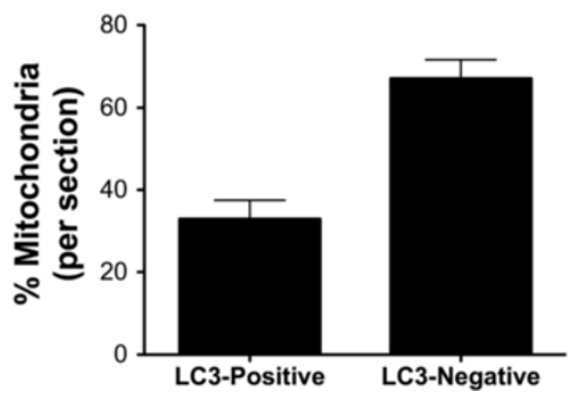

Figure 4 Vesicles forming from mitochondria are autophagosomes. A. LCC9 cells were transfected with GFP-LC3, treated with $0.1 \%$ ethanol vehicle, $500 \mathrm{nM} \mathrm{ICl}$, ERa shRNA, or $10 \mu \mathrm{M}$ Imatinib for 24 hours and counterstained with DAPI. Confocal microscopy was used to determine LC3-positive puncta formation. B. LCC9 cells were treated with $0.1 \%$ ethanol vehicle, $100 \mathrm{nM} \mathrm{ICI}$, ERa shRNA, or $10 \mu \mathrm{M}$ Imatinib for 72 hours and protein isolated. Western blot hybridization was used to determine LC3-I/LC3-II and p62 levels. C. LCC9 cells were treated with vehicle, $500 \mathrm{nM} \mathrm{ICl}$, serum starvation, $2 \mathrm{ng} / \mathrm{mL}$ tunicamycin, $10 \mu \mathrm{M}$ Imatinib, or transfected with ATG7 siRNA, PARK2 (parkin) siRNA, or ERa shRNA for 24 hours. Autophagosome (modified monodansylcadaverine) and mitochondria (MitoTracker-GFP) fold change was determined by flow cytometry. D. LC3-immunogold EM of LCC9 cells. M indicates mitochondria; Av indicates an autophagic vesicle; * indicates mitochondria-autophagosome interaction; arrows indicate LC3-immunogold particles. E. Mitochondria were counted and represented as percent of mitochondria either labeled with LC3-immunogold or unlabeled. $n=3-4,{ }^{*} p<0.05$. 
with serum starvation, $500 \mathrm{nM} \mathrm{ICI}, 2 \mathrm{ng} / \mathrm{mL}$ tunicamycin (as a positive control), ATG7 siRNA (as a negative control), $\mathrm{ER} \alpha$ shRNA, or $10 \mu \mathrm{M}$ Imatinib for 24 hours to increase autophagy. Prior to cell harvesting, cells were incubated with lentiviral MitoTracker-GFP. Cells were collected and treated with a modified monodansylcadaverine and sorted by flow cytometry to quantify autophagosome and mitochondria number (Figure 4C). Serum starvation, ICI, tunicamycin, ER $\alpha$ shRNA, or Imatinib treatment resulted in increased autophagosome formation. Serum starvation, ICI, tunicamycin, and ER $\alpha$ shRNA decreased overall mitochondrial content when compared with vehicle treated control. Imatinib had no significant effect on mitochondrial content; consistent with the maintenance of p62 levels indicating that autophagic flux is likely inhibited by drug treatment.

Transfection of LCC9 cells with ATG7 siRNA to inhibit autophagy reduced basal autophagosome formation with no significant change in mitochondrial flux. Inhibition of mitophagy, via parkin knockdown, also inhibited mitochondrial flux with no effect on autophagosome formation. These data indicate a reciprocal relationship between autophagy and mitochondria, suggesting either that mitochondria are the cellular content of autophagosomes (mitophagy) and/or that mitochondria are being used as the "raw material" to form autophagosomes as we observe in Figure 2. Furthermore, co-localization of GFPLC3 with Mitotracker-RFP, GolgiTracker-RFP, or EndoTracker (endoplasmic reticulum dye) was determined by confocal microscopy (Figure 5). We show that LC3 predominately localizes with the mitochondria. Moreover, images from LCC9 cells incubated with LC3-immunogold and studied by electron microscopy show that LC3 is localized to vesicles forming from mitochondria (Figure 4D), supporting the interpretation that the vesicles developing from mitochondria are autophagosomes. Quantification of the LC3-immunogold EM staining shows that the percentage of mitochondria per section that stain positive for
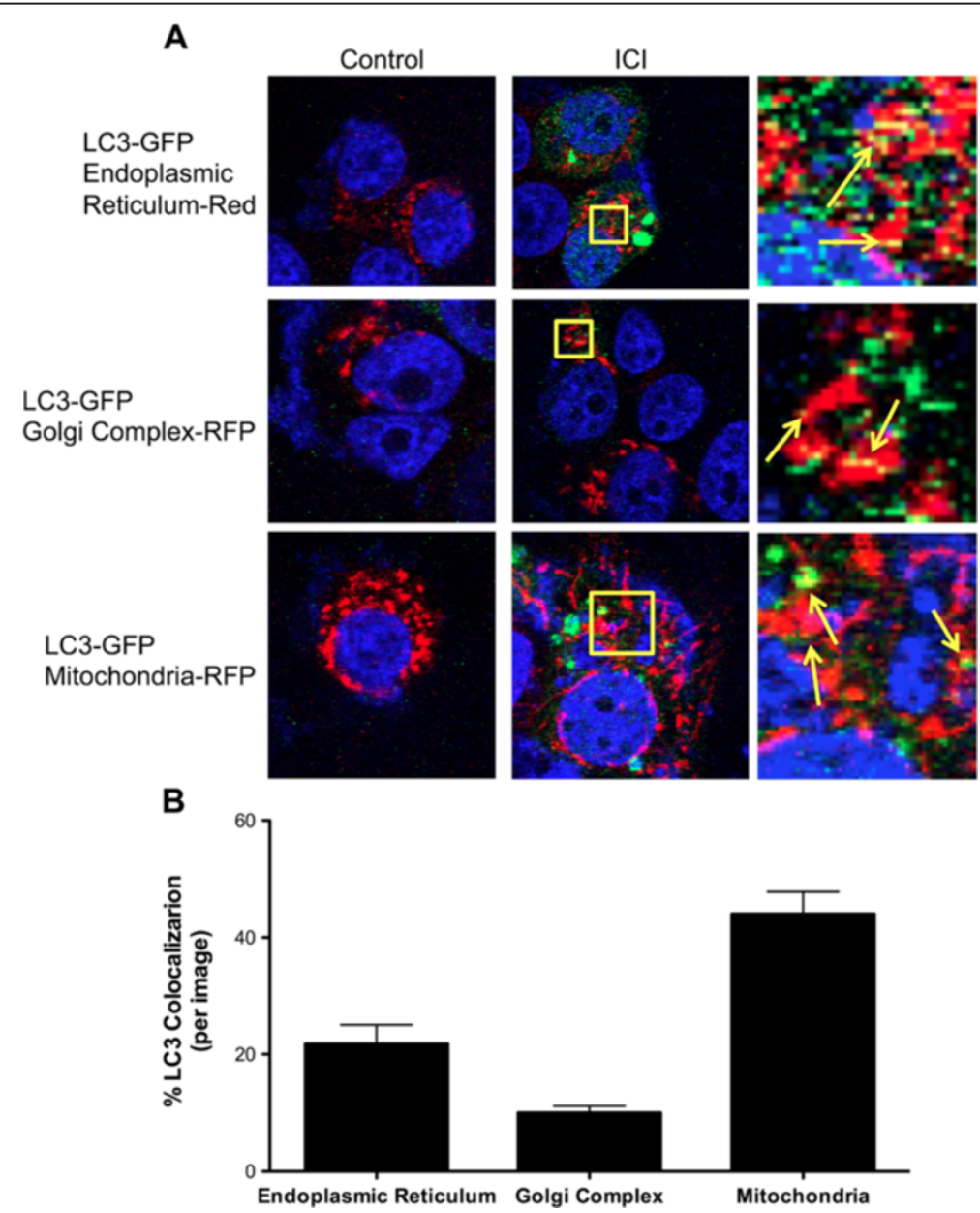

Figure 5 Co-localization of autophagosomes and cellular organelles. A. LCC9 cells were transfected with GFP-LC3 for 24 hours. Cells were incubated with either endoplasmic reticulum dye, Golgi complex-RFP, or MitoTracker-RFP and treated with $500 \mathrm{nM} I \mathrm{ICI}$ to induce autophagy. Confocal microscopy was used to determine the co-localization of LC3 puncta with different cellular organelles. B. The percent of LC3-positive autophagosome co-localizing with different cellular structures was determined. $n=5-6$. 
LC3 is approximately 35\% (Figure 4E). The percentage of mitochondria forming vesicles (Figure 3C) and the percentage of mitochondria stained positive for LC3 (Figure 4E) are similar, further supporting the likelihood that vesicles forming from mitochondria are autophagosomes. Data obtained by confocal microscopy confirm that these treatments induce autophagy, the flow cytometry data reflects both autophagosome and mitochondria flux, and the EM images show that mitochondrial membranes contribute to the formation of a membrane encapsulated autophagosomal-like vesicle, most likely reflecting the recycling of damaged or unnecessary mitochondria to form autophagosomes.
Lastly, we investigated whether the mitochondriaforming autophagosomes may be a form of mitophagy. LCC9 cells were treated with vehicle control or $100 \mathrm{nM}$ ICI for 72 hours. Mitochondrial or cytoplasmic protein fractions were collected and western blot hybridization performed to determine PINK1, parkin, COX-IV (mitochondrial control), or $\beta$-tubulin (cytoplasmic control). Treatment with ICI increased both PINK1 and parkin localization to the mitochondria (Figure 6A). Moreover, inhibition of mitophagy through PINK1 knockdown resensitized LCC9 cells to antiestrogen therapy, suggesting a dependence of LCC9 cells on functional mitophagy to maintain an antiestrogen resistant phenotype (Figure 6B).
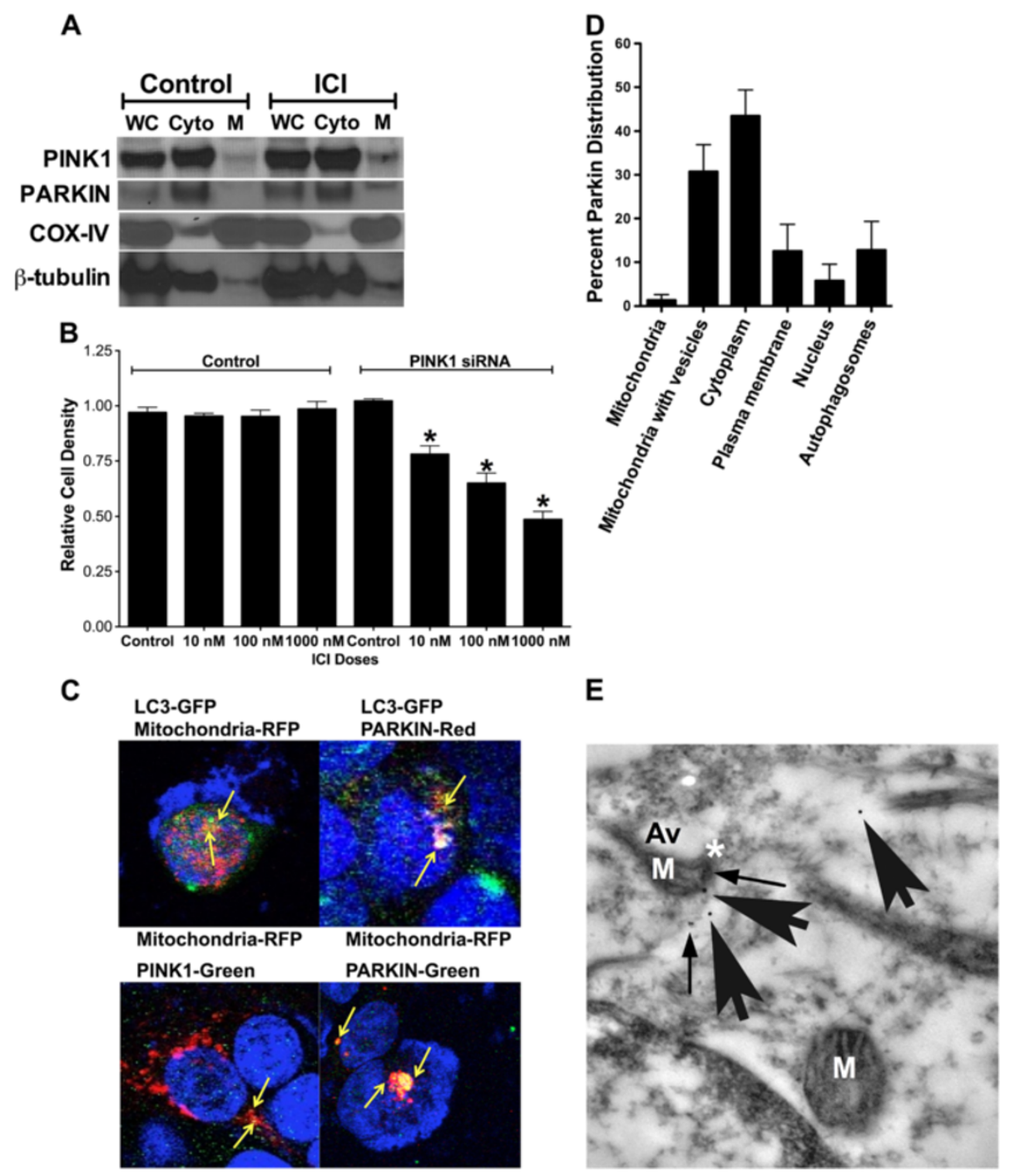

Figure 6 Autophagosomes forming from mitochondria is a novel form of parkin-associated mitophagy. A. Distribution of parkin and PINK1 in mitochondrial and cytoplasmic fractions of control and fulvestrant treated LCC9 cells as determined by western blot hybridization. B. Effect of mitophagy inhibition by PINK1 knockdown on antiestrogen sensitivity in LCC9 cells. C. Confocal microscopy of PINK1, parkin, LC3, and mitochondria in LCC9 cells treated with $100 \mathrm{nM} \mathrm{ICI} \mathrm{for} 72$ hours. D. Quantification of parkin-immunogold EM parkin distribution within LCC9 cells. $n=5$. E. EM image of parkin-immunogold stained LCC9 cells. Mitochondria forming vesicles stain positive for parkin. M indicates mitochondria: Av indicates an autophagic vesicle; * indicates mitochondria-autophagosome interaction; arrows indicate parkin-immunogold particles. 
The antiestrogen resistant LCC9 human breast cancer cells exhibit an elevated level of endogenous parkin expression when compared with their endocrine sensitive parental cell line (data not shown), further supporting an important role of mitophagy in antiestrogen responsiveness. Additional studies into the mechanistic contribution of mitophagy to antiestrogen resistance are ongoing.

Confocal microscopy was performed on LCC9 cells treated with $100 \mathrm{nM}$ ICI and either transfected with GFP-LC3 or incubated with a PINK1 antibody, parkin antibody, or mitotracker-RFP. As shown in Figure 6C when mitophagy is stimulated by ICI treatment, mitochondria localize with LC3, PINK1, and parkin. Moreover, LC3 also co-localizes with parkin, suggesting that mitochondria labeled with parkin are then either used to form autophagosomes or are engulfed by the forming autophagosomes. EM images suggest that both processes occur in ICI treated LCC9 cells; Figure 2 shows autophagosomes forming from mitochondria membranes, while Figure 7B shows an example of classical mitophagy where a mitochondria is localized inside a formed autophagosome. LCC9 cells were incubated with parkin-immunogold, and subsequent electron microscopy showed that parkin localized to mitochondria forming autophagosomes (Figure 6D). Thus, autophagosomes developing from mitochondria appear to represent a novel mechanism of mitophagy. Cellular parkin distribution is shown in Figure 6E, with parkin predominately localized within the cytoplasm and at mitochondria forming autophagosomes.
Autophagy is thought to occur naturally in most cells, and breast cancer cells often exhibit increased autophagy when compared with immortalized normal breast epithelial cells. Antiestrogen resistant breast cancer cells exhibit a further increase in autophagy when compared with their therapy sensitive counterparts $[15,19,20]$. We cannot exclude the possibility that these higher levels of autophagy in cancer cells result in the use of cellular materials or processes not commonly used in normal cells. Nonetheless, the use of preexisting target organelle membranes is an energy efficient process compared with de novo biosynthesis of a new double membrane, particularly if the membrane is at least partly obtained from the organelle being targeted for later degradation in the mature autolysosome. Moreover, we show that the process of mitochondrial-mediated autophagosome formation also occurs in MCF7 cells (ER+, antiestrogen sensitive breast cancer cells), implying that this phenomenon occurs more broadly than in just the LCC9 variant (Figure 7A). Since autophagy clearly plays an important role in breast cancer progression and therapeutic responsiveness [12,21,22], understanding how autophagy occurs may improve our ability to efficiently target this prosurvival pathway.

In conclusion, we show the first physical evidence, by electron microscopy, that mitochondria can supply membrane material during the creation of autophagosomes. We demonstrate that this occurs not only during serum starvation [8], but also during both basal (in the presence of serum and vehicle) and drug-induced autophagy. We
A

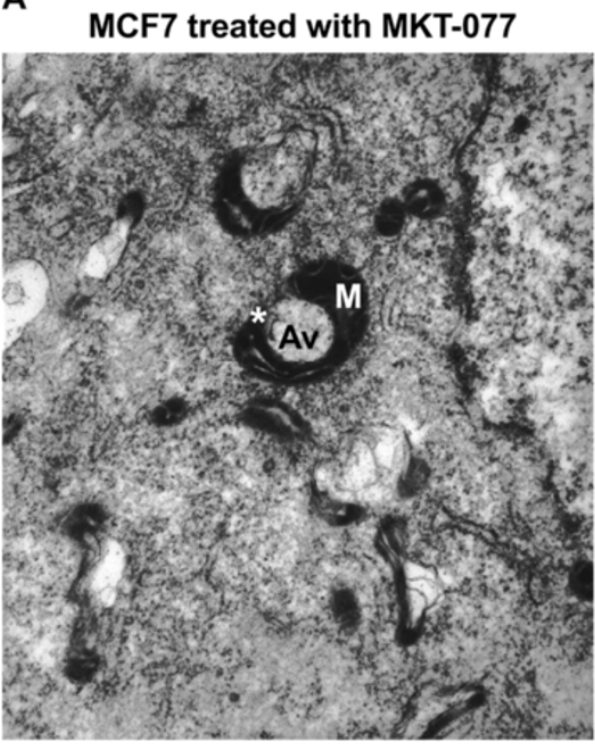

B

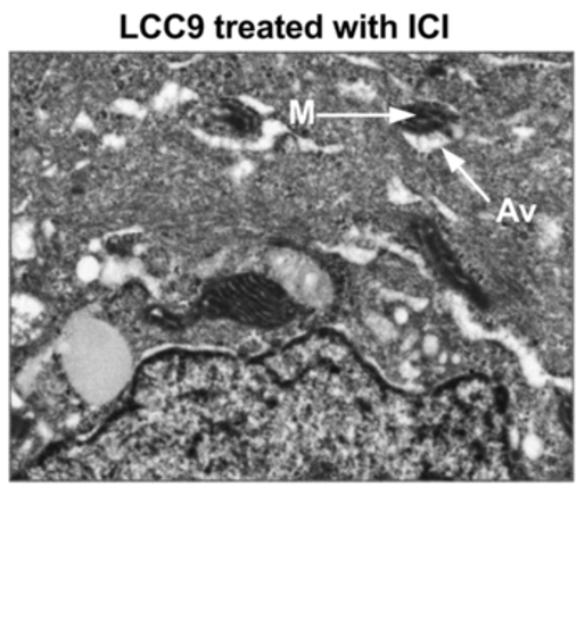

Figure 7 Mitophagy in human breast cancer cells. A. EM image of MCF7 cells treated with MKT-077, a cationic drug that concentrates in the mitochondria and inhibits glucose-regulated protein 75. Image indicates that mitochondria forming autophagosomes occur in other cell lines. B. EM image of LCC9 cells treated with ICI. Image shows an example of classical mitophagy where a mitochondria is engulfed by an autophagosome. 
go further to demonstrate that the autophagosomes developing from mitochondria may represent a novel mechanism of parkin-associated mitophagy, where mitochondrial membrane material can be contributed to formation of the developing autophagosome, rather than the autophagosome forming around parkin-labeled mitochondria. While we did not find similar early structures for autophagosomes incorporating other subcellular organelles, the data imply that the autophagic removal of Golgi/secretory vacuoles (crinophagy), endoplasmic reticulum (reticulophagy), and other organelles may also proceed with the contribution of target organelle membrane to formation of the membranes of the subsequent autophagosomes.

\section{Abbreviations}

ANOVA: Analysis of variance; ATG5: Autophagy related gene 5;

ATG7: Autophagy related gene 7; CCS: Charcoal stripped calf serum;

DAPI: 4',6-diamidino-2-phenylindole; EM: Electron microscopy; ER: Estrogen receptor; GFP: Green fluorescent protein; ICl: Faslodex, fulvestrant, ICI 182,780; LC3: Microtubule-associated protein light chain 3; PARK2: Parkin; RFP: Red fluorescent protein; SEM: Standard error of the mean; TAM: Tamoxifen.

\section{Competing interests}

Authors have no competing interests to declare.

\section{Authors' contributions}

KLC performed experiments, analyzed data, and wrote the manuscript. DSP, $M A$, and PAGC performed experiments and edited the manuscript. DDR and RC wrote and edited the manuscript. All authors read and approved the final manuscript.

\section{Acknowledgements}

Katherine Cook is supported by a DOD Breast Cancer Research Program Postdoctoral Fellowship (BC112023). This research was also supported in part by awards from the US Department of Health and Human Services (R01-CA131465 and U54-CA149147) to Robert Clarke and from the Intramural Research Program of the $\mathrm{NIH} / \mathrm{NCl}$ to David Roberts.

\section{Author details}

${ }^{1}$ Department of Oncology and Lombardi Comprehensive Cancer Center, Georgetown University Medical Center, Washington, DC 20057, USA. ${ }^{2}$ Laboratory of Pathology, Center for Cancer Research, National Cancer Institute, National Institutes of Health, Bethesda, MD 20892, USA. ${ }^{3}$ Immunopathology Section, Laboratory of Immunology, National Eye Institute National Institutes of Health, Bethesda, MD 20892, USA

Received: 13 November 2013 Accepted: 6 February 2014 Published: 27 March 2014

\section{References}

1. He C, Klionsky DJ: Regulation mechanisms and signaling pathways of autophagy. Annu Rev Genet 2009, 43:67-93.

2. Mari M, Tooze SA, Reggiori F: The puzzling origin of the autophagosomal membrane. F1000 biology reports 2011, 3:25

3. Rubinsztein DC, Shpilka T, Elazar Z: Mechanisms of autophagosome biogenesis. Current biology: CB 2012, 22:R29-R34

4. Dunn WA Jr: Studies on the mechanisms of autophagy: formation of the autophagic vacuole. J Cell Biol 1990, 110:1923-1933.

5. Dunn WA Jr: Studies on the mechanisms of autophagy: maturation of the autophagic vacuole. J Cell Biol 1990, 110:1935-1945.

6. Hayashi-Nishino M, Fujita N, Noda T, Yamaguchi A, Yoshimori T, Yamamoto A: A subdomain of the endoplasmic reticulum forms a cradle for autophagosome formation. Nat Cell Biol 2009, 11:1433-1437.

7. Luo S, Chen Q, Cebollero E, Xing D: Mitochondria: one of the origins for autophagosomal membranes? Mitochondrion 2009, 9:227-231.
8. Hailey DW, Rambold AS, Satpute-Krishnan P, Mitra K, Sougrat R, Kim PK, Lippincott-Schwartz J: Mitochondria supply membranes for autophagosome biogenesis during starvation. Cell 2010, 141:656-667.

9. McEwan DG, Dikic I: Not all autophagy membranes are created equal. Cell 2010, 141:564-566.

10. Jin SM, Youle RJ: PINK1- and Parkin-mediated mitophagy at a glance. J Cell Sci 2012, 125:795-799.

11. Soto-Pantoja DR, Miller TW, Pendrak ML, DeGraff WG, Sullivan C, Ridnour LA, Abu-Asab M, Wink DA, Tsokos M, Roberts DD: CD47 deficiency confers cell and tissue radioprotection by activation of autophagy. Autophagy 2012, 8:1628-1642.

12. Cook KL, Shajahan AN, Clarke R: Autophagy and endocrine resistance in breast cancer. Expert Rev Anticancer Ther 2011, 11:1283-1294.

13. Clarke R, Shajahan AN, Riggins RB, Cho Y, Crawford A, Xuan J, Wang Y Zwart A, Nehra R, Liu MC: Gene network signaling in hormone responsiveness modifies apoptosis and autophagy in breast cancer cells. J Steroid Biochem Mol Biol 2009, 114:8-20.

14. Schoenlein PV, Periyasamy-Thandavan S, Samaddar JS, Jackson WH, Barrett JT: Autophagy facilitates the progression of ERalpha-positive breast cancer cells to antiestrogen resistance. Autophagy 2009, 5:400-403.

15. Cook KL, Shajahan AN, Warri A, Jin L, Hilakivi-Clarke LA, Clarke R: Glucose-regulated protein 78 controls cross-talk between apoptosis and autophagy to determine antiestrogen responsiveness. Cancer Res 2012, 72:3337-3349.

16. Samaddar JS, Gaddy VT, Duplantier J, Thandavan SP, Shah M, Smith MJ, Browning D, Rawson J, Smith SB, Barrett JT, Schoenlein PV: A role for macroautophagy in protection against 4-hydroxytamoxifen-induced cell death and the development of antiestrogen resistance. Mol Cancer Ther 2008, 7:2977-2987.

17. Brunner N, Boysen B, Jirus S, Skaar TC, Holst-Hansen C, Lippman J, Frandsen T, Spang-Thomsen M, Fuqua SA, Clarke R: MCF7/LCC9: an antiestrogen-resistant MCF-7 variant in which acquired resistance to the steroidal antiestrogen ICI 182,780 confers an early cross-resistance to the nonsteroidal antiestrogen tamoxifen. Cancer Res 1997, 57:3486-3493.

18. Ertmer A, Huber V, Gilch S, Yoshimori T, Erfle V, Duyster J, Elsasser HP, Schatzl HM: The anticancer drug imatinib induces cellular autophagy. Leukemia 2007, 21:936-942.

19. Cook KL, Clarke R: Heat shock $70 \mathrm{kDa}$ protein 5/glucose-regulated protein 78 "AMP"ing up autophagy. Autophagy 2012, 8:1827-1829.

20. Tu YF, Kaipparettu BA, Ma Y, Wong $\sqcup$ : Mitochondria of highly metastatic breast cancer cell line MDA-MB-231 exhibits increased autophagic properties. Biochim Biophys Acta 1807, 2011:1125-1132.

21. Vazquez-Martin A, Oliveras-Ferraros C, Menendez JA: Autophagy facilitates the development of breast cancer resistance to the anti-HER2 monoclonal antibody trastuzumab. PLoS One 2009, 4:e6251.

22. Thomas S, Thurn KT, Bicaku E, Marchion DC, Munster PN: Addition of a histone deacetylase inhibitor redirects tamoxifen-treated breast cancer cells into apoptosis, which is opposed by the induction of autophagy. Breast Cancer Res Treat 2011, 130:437-447.

doi:10.1186/2045-3701-4-16

Cite this article as: Cook et al:: Mitochondria directly donate their membrane to form autophagosomes during a novel mechanism of parkin-associated mitophagy. Cell \& Bioscience 2014 4:16.

\section{Submit your next manuscript to BioMed Central and take full advantage of:}

- Convenient online submission

- Thorough peer review

- No space constraints or color figure charges

- Immediate publication on acceptance

- Inclusion in PubMed, CAS, Scopus and Google Scholar

- Research which is freely available for redistribution 